\title{
Der Psycho-Boom (xinli re) in China
}

Konrad Michel

* www.ifp.name

Korrespondenz:

Dr. med. Konrad Michel Facharzt für Psychiatrie und Psychotherapie Bälliz 12

CH-3600 Thun

konrad.michel[at]spk.unibe.ch

\section{Die IFP und ihre Entwicklung}

Die IFP* entwickelte sich aus der 1934 gegründeten International General Medical Society for Psychotherapy (IGMSP), deren erster Präsident C.G. Jung war. Im Jahre 1958 wurde sie umbenannt in Federation for Medical Psychotherapy (IFMP), mit Medard Boss als Präsident. Unter Edgar Heim, Präsident von 1988 bis 1999, wurde die Bezeichnung «International Federation for Medical Psychotherapy» in «International Federation for Psychotherapy» abgeändert. Gemäss Prof. Edgar Heim: um die Aktivitäten auch auf osteuropäische Länder und Asien auszurichten. Eines der Ziele der IFP heisst: «The IFP encourages and supports development within psychotherapy corresponding to the specific requirements and necessities of the various continents, regions and cultures.» So fand 1994 der erste Kongress in Seoul, Korea, statt, unter dem Titel «Psychotherapy: East \& West, Integration of Psychotherapy». Es entstanden regionale «Chapters» der IFP, und regionale Tagungen wurden organisiert. Der letzte Weltkongress der IFP ging 2010 in Luzern über die Bühne.

\section{Der Kongress in Shanghai}

In Shanghai nun waren für die Organisation zuständig Zhao Xudong, Tongji University of Shanghai, Franz Caspar, Präsident der IFP, Universität Bern, sowie Xu Yifeng, Shanghai Mental Health Center. Die Grösse des Kongresses war eindrücklich: ca. 1200 Teilnehmer, fast alles Chinesen, wir Westler darunter waren klare Ausnahmen. Das Interesse an der Psychotherapie in China scheint gross, ja riesig zu sein.

Die drei Kongresstage waren vormittags Plenarvorträgen gewidmet, an den Nachmittagen fand eine Vielzahl von Parallelsymposien und Workshops statt. Auffallend viele Vorträge befassen sich mit der Geschichte der westlichen Geisteswissenschaften, speziell der Philosophie, Psychologie und Psychotherapie. Das Book of Abstracts liest sich wie ein Überblick über die Geschichte der europäischen, vor allem der deutschsprachigen Psychologie und Psychotherapie: Beim Blättern stösst man auf Vorträge und Symposien zu Namen wie Carl Jaspers, Sigmund Freud, C.G. Jung, Medard Boss (Daseinsanalyse und Taoismus).

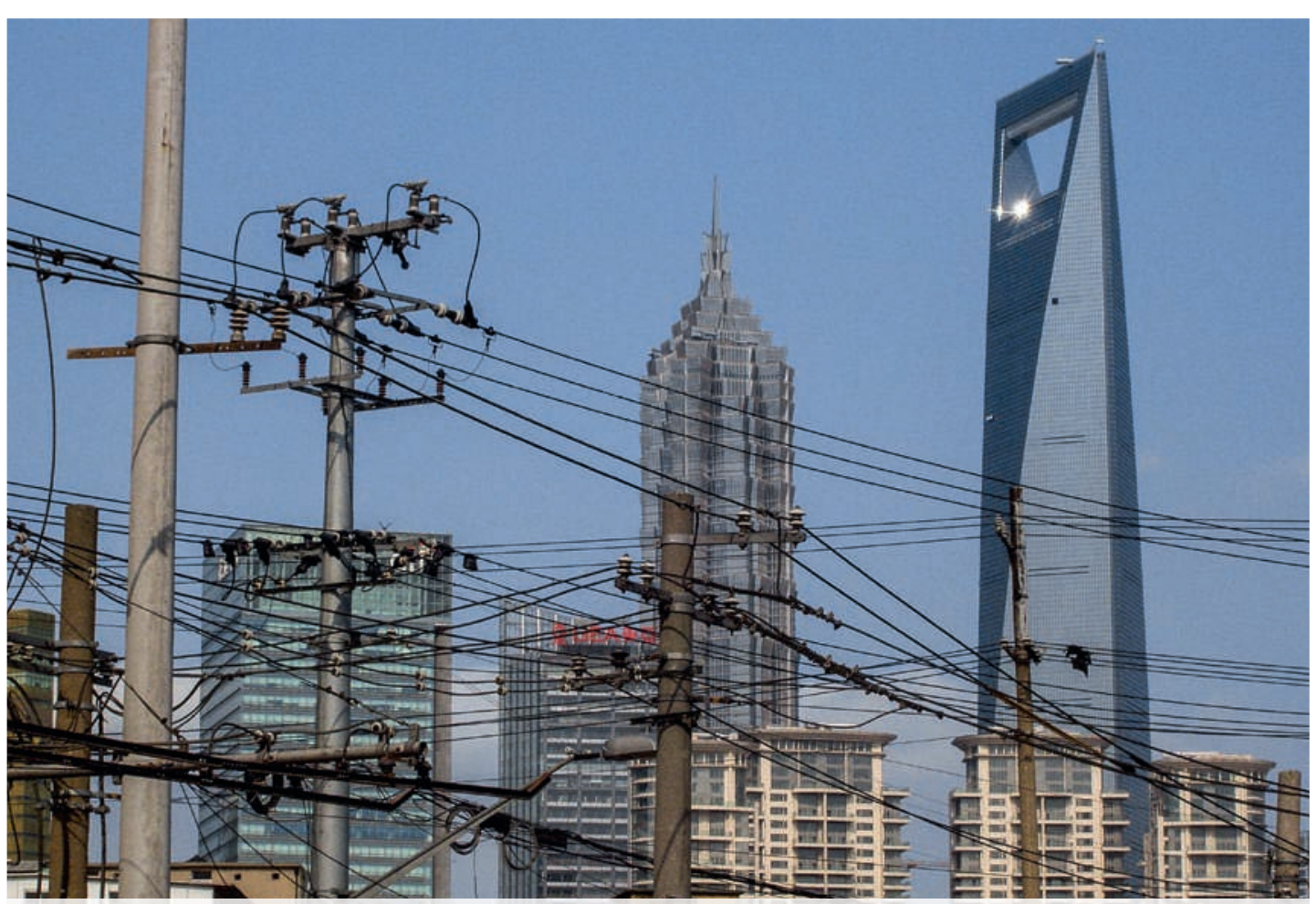

Die 23-Millionen-Metropole Shanghai war Schauplatz des 21. IFP-Kongresses. Nicht alles hält mit dem rasanten Wachstum Schritt. 


\section{Brücken zwischen zwei Welten schlagen}

Konzepte und psychotherapeutische Schulen werden in China nicht ohne Reflexion übernommen, sondern aus der Sicht der chinesischen Kultur analysiert und diskutiert, wobei meist versucht wird, eine Brücke zwischen den beiden Welten zu schlagen. Oft werden auch Synergien diskutiert, die sich aus der Psychologie der Traditionellen Chinesischen Medizin (TCM) mit der Öffnung gegenüber westlichen Konzepten ergeben. So ist die Rede von «Rebirth of Psychoanalysis in China» als eines der historischen Ereignisse, welches mitgeholfen hat, die chinesische Gesellschaft zu verändern. Zitat: «Psychoanalysis is part of human history and continues to get inscribed in it. Like the radiant Phoenix, this rebirth and new destiny in China may be ‘ a new beginning for Psychoanalysis that could in turn feed from Chinese ancestral cultural richness» (Zhang Tianbu und andere). Spannend sind die Reflexionen über Schopenhauer und Nietzsche aus chinesischer Sicht, die auch heute noch geprägt ist durch Konfuzius und die hohen moralischen Ansprüche an das Individuum. Meist wird eher das Verbindende als das Gegensätzliche betont, wie zum Beispiel
Auswuchs bürgerlicher Psychologie und Psychotherapie» bezeichnet wurde, kam es 1988, rund 10 Jahre nach dem Ende der Kulturrevolution, in Kunming zum ersten Deutsch-Chinesischen Kongress für Psychotherapie.

Es folgten weitere Kongresse sowie fortlaufende Seminare in psychoanalytisch orientierter Psychotherapie in Zusammenarbeit mit dem Shanghai Mental Health Center. Einen grossen Stellenwert bekam dabei die Aufarbeitung der - bloss zwei Generationen zurückliegenden - schwersten Traumatisierung eines grossen Teils der chinesischen Bevölkerung während der katastrophalen Kulturrevolution, in der Familien auseinandergerissen, Menschen verhaftet, gefoltert, gedemütigt wurden. Millionen von Menschen kamen ums Leben, dies, nachdem schon der vorangehende «Grosse Sprung nach vorne» an die 20 Millionen Hungertote gefordert hatte. Die Schwierigkeiten in der Bearbeitung der Folgen dieser Traumatisierungen über Generationen hinweg wird im sehr empfehlenswerten Buch ${ }^{* *}$ von Antje Haag (2011) eindrücklich beschrieben. Die Aufarbeitung fehlt in China nach wie vor praktisch vollkommen, und die Meinungen

\section{Konzepte und psychotherapeutische Schulen werden in China nicht ohne Reflexion übernommen, sondern aus der Sicht der chinesischen Kultur analysiert und diskutiert.}

auch in der Gegenüberstellung von Taoismus und Psychoanalyse.

Achtsamkeit ist ein Thema, Gestalttherapie als Verbindung von Ost und West, wie auch die integrierte Körpertherapie Qigong. Die narrative Therapie als «nicht-pathologisierende Technik» wird propagiert, was aber nicht wie bei uns im Sinne einer Anerkennung und Unterstützung der Autonomie des Individuums zu verstehen ist, sondern als «ways of being with others». Denn, und das wird zumindest in einem Symposium zur Psychologie der TCM betont, das Ziel einer Therapie ist, das Selbst aufzulösen, wobei allerdings betont wird, dass man gegen aussen auch eine - fassbare - Form (shape) braucht. Im Weiteren finden sich auch ziemlich exotische Therapieformen wie «Mountain Stream Therapy», Erwärmen der Hände im Wasser, und chinesische Kalligraphie (Xie Yi painting) als Form von Psychotherapie.

Deutsch-chinesische Seminare für Psychotherapie Spannend war das Symposium zur «Transgenerational Transmission of Trauma», wobei die drei Referate ausschliesslich von deutschen Kollegen bestritten wurden, mit einer grossen chinesischen Zuhörerschaft. Die deutsch-chinesischen Seminare für Psychotherapie haben Tradition. Nachdem die Psychoanalyse seit Gründung der Volksrepublik 1949 als «schädlichster in der heutigen Gesellschaft zur chinesischen Geschichte des 20. Jahrhunderts sind stark durch die offizielle Geschichtsschreibung geprägt, die sich bis jetzt noch überhaupt nicht von Chairman Mao Zedong distanziert hat.

Ulrich Schnyder, Zürich, stellte die evidenzbasierten Traumatherapien vor. Interessant ist, dass in China EMDR eine wichtige Rolle spielt, möglicherweise weil es ein überblickbares Therapieprogramm ist mit dem Ziel, die gesundheitlichen Auswirkungen von Traumatisierungen zu neutralisieren. Die erstaunlich wirksame «Well-Being Therapy» der Mailänder Gruppe von Giovanni Fava und Mitarbeitern aus Mailand würde sich wohl ebenfalls für China eignen. Mehr als einmal hörte ich, dass - da sich Probleme häufig nicht lösen lassen - das Ziel einer Therapie sein müsse, mit bzw. trotz den Problemen besser leben zu können. Generell bekam ich den Eindruck, dass die traditionelle chinesische Kultur dem analytischen Aufdecken grundsätzlich abgeneigt und mehr auf das Überwinden von Problemen und das Finden von neuer Harmonie ausgerichtet ist. Dazu gehört auch das Stichwort Resilienz - angesichts der Spontaneität und der Herzlichkeit, die der westliche Besucher in China antrifft, würde ich annehmen, dass die chinesische Gesellschaft hohe Werte auf einer Resilienzskala aufweist. 
Liu Tianjun, Präsident des Center for Traditional Chinese Medicine in Beijing, war mit seiner provokativen Gegenüberstellung von chinesischer und westlicher Psychologie eher eine Ausnahme. Psychologie an sich sei ein westliches Konzept, in der traditionellen chinesischen Kultur seien mind und body eine Einheit (Tao). Dies im Gegensatz zum Westen, wo es darum gehe, das Selbst zu stärken, um mit Problemen umgehen zu können, während in China das Selbst als Problem an sich gesehen werde, weil es die Ursache der Probleme sei. Logischerweise ist das Ziel der Therapie, das Selbst aufzulösen, ja abzuschaffen. «When we overcome the self, we overcome all problems.» Barbara Hofmann-Huber aus Deutschland, selber Qigong-Therapeutin, stellte richtig, dass Liu's Konzept des Selbst im psychoanalytischen Modell eher unserem Ego entsprechen würde, und dass die beiden Konzepte nicht unvereinbar sind. Hier kam das Verbindende zur Abwechslung aus dem Westen.

Generell leuchtete das Thema des Konflikts zwischen Individuation und Anpassung (Kollektivismus) im Kongress immer wieder auf. In der chinesischen Kultur galt (gilt?) es als schlecht und gefährlich, die eigenen Interessen in den Vordergrund zu stellen. Die Loyalität gegenüber der Gesellschaft, und vor allem gegenüber der Familie hatte - und hat auch heute noch - Priorität.

\section{Klinische Psychologie vorwiegend an} universitären Zentren

In der Praxis ist es so, dass Klinische Psychologie, wenn überhaupt, vor allem in einigen universitären Zentren existiert. In den grossen Kliniken und den in den Städten bestehenden Mental-Health-Zentren scheint es bis jetzt kaum Psychologen zu geben. Seit

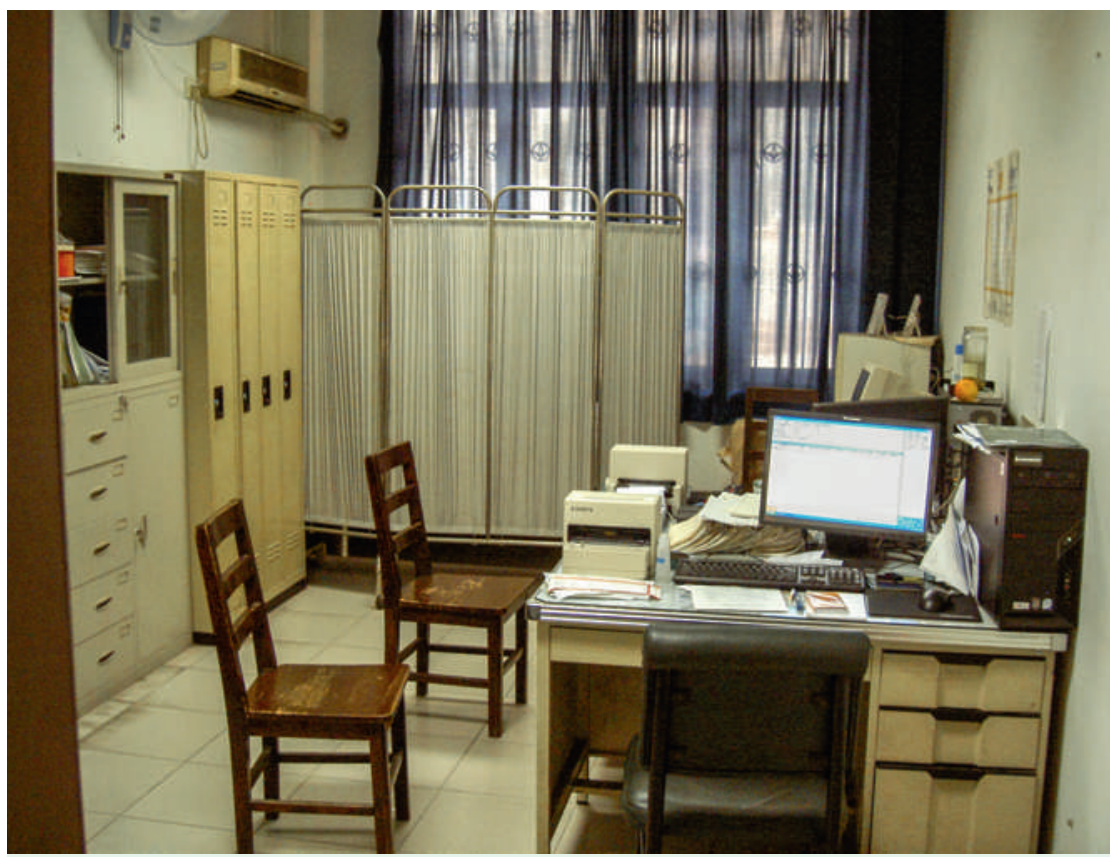

Ambulante Psychiatrie im nicht-universitären Xuhui Mental Health Center: Zwei Ärzte sehen ihre Patienten gleichzeitig zu 5-Minuten Konsultationen im selben Raum. dem 1. Mai 2013 ist das neue Mental Health Law in Kraft, ein komplexes Gebilde, aber zumindest kommen Psychotherapie und psychologische Beratung neu als gesetzlich verankerte Behandlungsmethoden darin vor. China mit seiner Bevölkerung von 1,34 Milliarden hat aktuell 20000 Psychiater und braucht in den nächsten Jahren 100000 (!) neue Psychiater und Therapeuten. Man sagte mir, dass - wie in Europa, wobei die Gründe wohl andere sind - immer weniger Ärzte Psychiatrie als Fach wählen. Über 80 Mio. Menschen leiden unter Depressionen und Angststörungen, über 180000 Menschen begehen jährlich Suizid, und 2 Mio. begehen Suizidversuche. Kein Wunder, dass Yu Xin, der junge Präsident des Bejing Mental Health Centers, angesichts der sehr beschränkten Ressourcen den Bedarf nach einfachen («simple and easy to learn») Therapiemethoden betonte.

Norman Sartorius, Genf, zeigte auf, wie prekär die Situation global ist: In drei Vierteln der Welt reduziert sich die Behandlung psychischer Erkrankungen auf Pharmakotherapie. Die Situation in den weitaus meisten chinesischen Kliniken und Mental Health Centers ist nicht anders. Ich hatte in den letzten Jahren die Gelegenheit, zahlreiche psychiatrische Kliniken im Land besuchen zu können: 5-Minuten-Konsultationen sind die Regel, praktisch durchwegs Pharmakotherapie. Ein Erstgespräch von 60 Minuten ist undenkbar. Psychotherapie wird offenbar fast ausschliess-

\section{Die Wirkung der Antidepressiva erklären sie ganz einfach mit «it will make you feel better».}

lich in den grossen universitären Zentren praktiziert und unterrichtet und hat in der Gesundheitsversorgung bis jetzt einen marginalen Stellenwert. Die Weiterbildung in der Psychiatrie schliesst zwar die Theorie der hauptsächlichen psychotherapeutischen Schulen ein, aber deren praktische Anwendung im Rahmen der Weiterbildung gibt es nicht, ebenso wenig wie eine Psychotherapie-Supervision.

$\mathrm{Ob}$ es China in den nächsten Jahren gelingen wird, die Psychotherapie im Land als Behandlungsform zu etablieren, weiss zurzeit wohl niemand. Das Interesse ist da, und der Bildungshunger offensichtlich gross. Unmöglich ist es nicht; in China scheint alles zehnmal schneller zu gehen als bei uns. Ein Hindernis könnte die in China nach wie vor stark verbreitete Stigmatisierung psychischer Gesundheitsprobleme sein. Psychiater erzählten mir, dass sie bei der Verschreibung von Antidepressiva das Wort Depression besser nicht in den Mund nehmen. Die Wirkung der Antidepressiva erklären sie ganz einfach mit «it will make you feel better». Da wird wohl noch einiges Wasser den Yangtse hinunterfliessen, bis auch die Psychotherapie der Depression - und anderer Störungen in der chinesischen Kultur Eingang gefunden hat. 